\title{
from headquarters
}

To communicate more efficiently with members and to provide better services, the AMS Headquarters recently became a full node on the Internet with the domain name ametsoc.org. The following lists all e-mail addresses and responsibilities for all Headquarters staff members and staff members at the Washington, D.C., office. A number of general addresses and their intended use are are also listed. In addition, while 617-2272425 continues to serve as the primary number for calls to AMS Headquarters, callers who know the extension of the party they wish to reach can call 617-227-2426 and enter the extension at the prompt. Extensions are included in the staff listing below. Any staff at the Washington, D.C., office can be reached at 202-466-6070.

\section{AMS Staff}

\begin{tabular}{ll}
\multicolumn{1}{c}{ Name } & Ext. \\
Irwin Abrams & 208 \\
Joyce Annese & 206 \\
Stephanie Armstrong & 235 \\
Elisabeth Bacon & 209 \\
Helen Beattie & 231 \\
Debbie Begreen & 227 \\
Bernard Blair & DC \\
Julie Burba & 241 \\
Rose Circeo & 216 \\
Jane Dannenberg & 200 \\
Regina Dobson & 228 \\
Patricia English & 229 \\
Donna Fernandez & 246 \\
Kelly Sheahan Garvey & 215 \\
Ira Geer & DC \\
David Gershman & 236 \\
Katy Ginger & DC \\
Todd Glickman & 237 \\
Claudia Gorski & 226 \\
Gary Gorski & 230 \\
Richard E. Hallgren & 201 \\
Bryan Hanssen & 238 \\
Beth Jarabek & 214 \\
Corinne Kazarosian & 217 \\
Michael Malta & 234 \\
Evelyn Mazur & 204 \\
Mary McMahon & 218 \\
Don McManus & DC \\
Barry Mohan & 205 \\
Greta Morris & 203 \\
Karen Morrissey & 226 \\
Stuart Muench & 302 \\
Annemarie Murphy & 211 \\
Harold Nagel & 223 \\
Keith Seitter & 220 \\
Yale Schiffman &
\end{tabular}

\section{E-mail}

iabrams@ametsoc.org jannese@ametsoc.org sarmstrg@ametsoc.org ebacon@ametsoc.org hbeattie@ametsoc.org dbegreen@ametsoc.org amsedu@cais.com jburba@ametsoc.org rcirceo@ametsoc.org jdannen@ametsoc.org rdobson@ametsoc.org penglish@ametsoc.org dfernand@ ametsoc.org kgarvey@ametsoc.org amsedu@cais.com dgershmn@ametsoc.org amsedu@cais.com glickman@ametsoc.org cgorski@ametsoc.org ggorski@ ametsoc.org hallgren@ametsoc.org bhanssen@ametsoc.org bjarabek@ametsoc.org ckazaros@ametsoc.org mmalta@ametsoc.org emazur@ametsoc.org mmcmahon@ametsoc.org amsedu@cais.com bmohan@ametsoc.org gmorris@ametsoc.org kmorrisy@ametsoc.org smuench@ametsoc.org amurphy@ametsoc.org halnagel@ametsoc.org kseitter@ametsoc.org yale@amsdc.mhs.

\section{Responsibility}

Technical Editor: BAMS, WAF Assistant to the Exec. Director Scholarship/Fellowships Member Services Copy Editor: JAM, JCLI AMS Meetings K-12 Education Programs News Editor: BAMS Network Administration Planned Giving AMS Meetings Senior Copy Editor: MWR, WAF Member Services Special Programs, Seal, CCM K-12 Program Director Production Manager: BAMS K-12 Education Programs Assistant Executive Director AMS Meetings Copy Editor: JAS Executive Director Production Assistant Customer Services MIS/Membership Manager Mail Center Operations Meetings Director Advertising/Page Charges K-12 Education Programs Controller Accounting

AMS Meetings Technical Editor: JAS, JCLI Assistant Controller Technical Editor: JAS, JCLI, JPO Associate Executive Director Director of Development 
Kenneth Spengler

Denise Taylor

Rachel Thomas

Monica Tolson

Kathleen Ventre

Melissa Weston

Kenneth Wilson

Stasia Zomkowski
DC

DC

250

242

233 spengler@ametsoc.org

dtaylor@ametsoc.org

rthomas@ametsoc.org

mtolson@amsdc.mhs.

compuserve.com

amsedu@ cais.com

mweston@ametsoc.org

kwilson@ametsoc.org

szomkows@ametsoc.org
Executive Director Emeritus

Copy Editor

Editorial Assistant

Exhibit Coordinator

K-12 Education Programs

Publications Coordinator

Technical Editor: JAM, JTECH, MWR

Copy editor: JTECH, JPO

\section{General E-mail Addresses}

amspubs@ametsoc.org amsempl@ametsoc.org amssubs@ametsoc.org amsmem@ametsoc.org amsmtgs@ametsoc.org amsaddr@ametsoc.org amsinfo@ametsoc.org scholar@ametsoc.org glossary@ametsoc.org chapnews@ametsoc.org amschap@ametsoc.org general information regarding publications inquiries/ads for employment announcements inquries about AMS subscriptions and publication pricing general inquiries about membership/Seal/CCM inquiries about meetings address changes for publication subscriptions general information about AMS inquires about scholarships/fellowships correspondence regarding new glossary chapter meeting minutes how to start a chapter; organizational and constitutional items

\section{Announcing The End Of The Ice Age.}

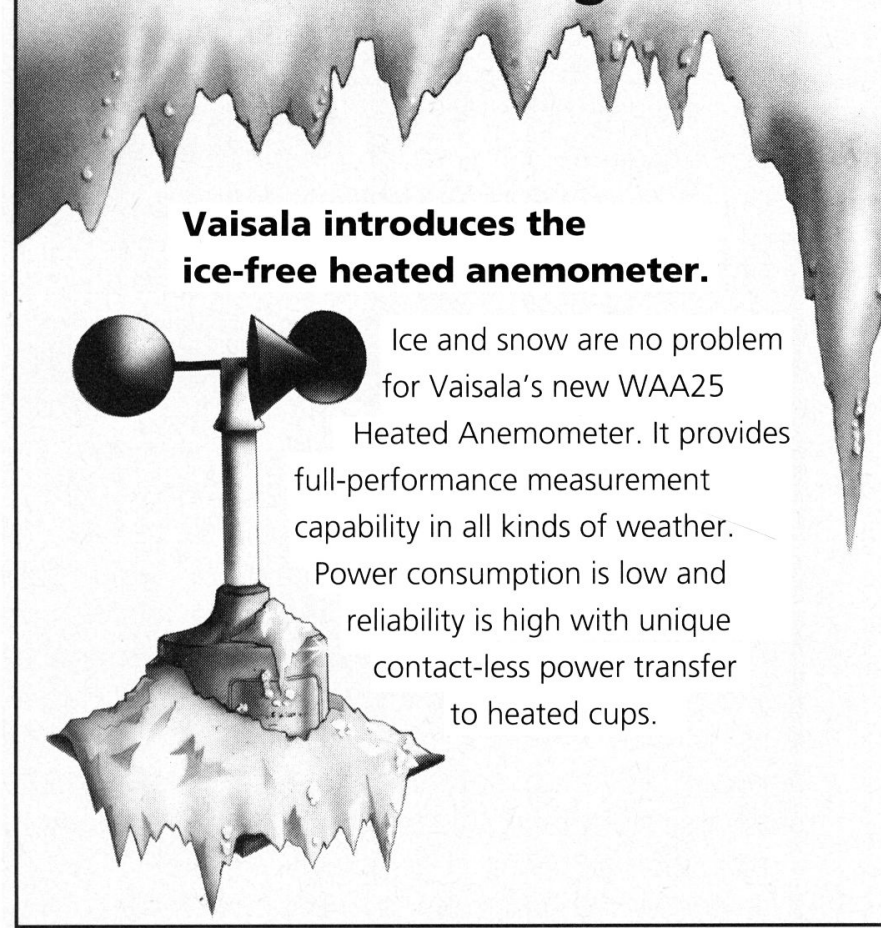

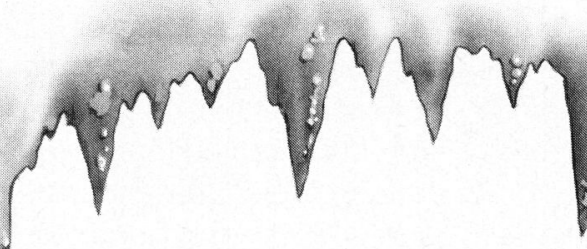

Free Literature. For more information on the new WAA25 Heated Anemometer and other ice-free wind instruments, call or fax Vaisala today. And make the problem of icing a thing of the past.

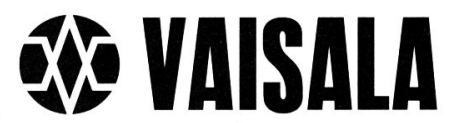

Measuring the Environment

100 Commerce Way, Woburn, MA 01801 Tel: (617) 933-4500 Fax: (617) 933-8029 